University of Nebraska - Lincoln

DigitalCommons@University of Nebraska - Lincoln

Mechanical \& Materials Engineering Faculty

Publications

Mechanical \& Materials Engineering,

Department of

\title{
$2-2018$
}

\section{Eigenstrain as a mechanical set-point of cells}

\author{
Shengmao Lin \\ University of Nebraska-Lincoln, linshengmao@gmail.com \\ Marsha C. Lampi \\ Cornell University \\ Cynthia A. Reinhart-King \\ Cornell University, cak57@cornell.edu \\ Gary C.P. Tsui \\ Hong Kong Polytechnic University, mfgary@polyu.edu.hk \\ Jian Wang \\ University of Nebraska-Lincoln, jianwang@unl.edu
}

\section{See next page for additional authors}

Follow this and additional works at: https://digitalcommons.unl.edu/mechengfacpub

Part of the Biomechanics and Biotransport Commons, Mechanics of Materials Commons, Molecular, Cellular, and Tissue Engineering Commons, Nanoscience and Nanotechnology Commons, Other Biomedical Engineering and Bioengineering Commons, Other Engineering Science and Materials Commons, and the Other Mechanical Engineering Commons

Lin, Shengmao; Lampi, Marsha C.; Reinhart-King, Cynthia A.; Tsui, Gary C.P.; Wang, Jian; Nelson, Carl A.; and Gu, Linxia, "Eigenstrain as a mechanical set-point of cells" (2018). Mechanical \& Materials Engineering Faculty Publications. 291.

https://digitalcommons.unl.edu/mechengfacpub/291

This Article is brought to you for free and open access by the Mechanical \& Materials Engineering, Department of at DigitalCommons@University of Nebraska - Lincoln. It has been accepted for inclusion in Mechanical \& Materials Engineering Faculty Publications by an authorized administrator of DigitalCommons@University of Nebraska Lincoln. 


\section{Authors}

Shengmao Lin, Marsha C. Lampi, Cynthia A. Reinhart-King, Gary C.P. Tsui, Jian Wang, Carl A. Nelson, and Linxia Gu 


\title{
Eigenstrain as a mechanical set-point of cells
}

\author{
Shengmao Lin,, Marsha C. Lampi, ${ }^{3}$ \\ Cynthia A. Reinhart-King, ${ }^{3,4}$ Gary Tsui, ${ }^{5}$ \\ Jian Wang, ${ }^{2}$ Carl A. Nelson, ${ }^{2}$ and Linxia $\mathrm{Gu}^{2}$
}

\author{
1 School of Civil Engineering and Architecture, Xiamen University of \\ Technology, Xiamen, China \\ 2 Department of Mechanical and Materials Engineering, University of \\ Nebraska-Lincoln, Lincoln, NE 68588-0656, USA \\ 3 Biomedical Engineering, Cornell University, Ithaca, NY 14853, USA \\ 4 Biomedical Engineering, Vanderbilt University, Nashville, TN 351631, USA \\ 5 Department of Industrial and Systems Engineering, The Hong Kong \\ Polytechnic University, Hung Hom, Kowloon, Hong Kong, People's Republic \\ of China \\ Corresponding author — Linxia Gu, email Igu@unl.edu
}

\begin{abstract}
Cell contraction regulates how cells sense their mechanical environment. We sought to identify the set-point of cell contraction, also referred to as tensional homeostasis. In this work, bovine aortic endothelial cells (BAECs), cultured on substrates with different stiffness, were characterized using traction force microscopy (TFM). Numerical models were developed to provide insights into the mechanics of cell-substrate interactions. Cell contraction was modeled as eigenstrain which could induce isometric cell contraction without external forces. The predicted traction stresses matched well with TFM measurements. Furthermore, our numerical model provided cell stress and displacement maps for inspecting the fundamental regulating mechanism of cell mechanosensing. We showed that cell spread area, traction force on a substrate, as well as the average stress of a cell were increased in response to a stiffer substrate. However, the cell average strain, which is cell type-specific, was kept at the same level regardless of the substrate stiffness. This indicated that the cell average strain is the tensional homeostasis that each type of cell tries to maintain. Furthermore,
\end{abstract}


cell contraction in terms of eigenstrain was found to be the same for both BAECs and fibroblast cells in different mechanical environments. This implied a potential mechanical set-point across different cell types. Our results suggest that additional measurements of contractility might be useful for monitoring cell mechanosensing as well as dynamic remodeling of the extracellular matrix (ECM). This work could help to advance the understanding of the cell-ECM relationship, leading to better regenerative strategies.

Keywords: Eigenstrain, Tensional homoeostasis, Steady state, Cell contraction, TFM, Mechanosensing

\section{Introduction}

The extracellular matrix (ECM) is essential for regulating cell behavior and tissue function (Discher et al. 2005; Oria et al. 2017). Local ECM structure and mechanics are increasingly recognized as important mechanical effectors of cell responses and tissue regeneration (Yu et al. 2011; Lin 2017). This is illustrated by the fact that both ECM rigidity (Hadjipanayi et al. 2009) and local tension (Beloussov et al. 2000) regulate cellular mechanotransduction pathways, and their dysregulation results in many different types of disease (Rittweger et al. 2009). Following mechanical disturbance of the ECM, cells can alter their contractile force and morphology including cell spread area, focal adhesion quantity and strength, and cytoskeleton structure (Califano and Reinhart-King 2010; Prager-Khoutorsky et al. 2011; Solon et al. 2007; Kumar et al. 2016). It has been speculated that cell contraction, generated by the cross-bridging interaction of actin and myosin II motors, maintains tensional homeostasis, i.e., a stable intracellular state, also referred as the set-point of cells (Paszek et al. 2005).

Various physical parameters, including tensional force on cells and cell stiffness, were proposed as the index factors of cellular tensional homeostasis (Brown et al. 1998; Mizutani et al. 2004). The early experiments illustrated that external loads led to an instantaneous alternation of cell behaviors including tensional force and stiffness. After unloading, cells returned to their original mechanical state. This indicated that cell stiffness and tensional force correlated with the magnitude of mechanical stimuli, instead of its loading history. However, the connection between different levels of mechanical stimuli, including different ECM stiffnesses, were not identified, which is the basis

for identifying the set-point of cells. Recent studies with traction force 
microscopy (TFM) suggest that cell geometry or strain energy could be a better index of tensional homeostasis (Califano and Reinhart-King 2010; Munevar et al. 2001; Oakes et al. 2014; Tan et al. 2003; Wang et al. 2002; Rape et al. 2011). However, integrating a variety of discrete experimental data to inspect the fundamental regulating factors of cells remains a challenge.

Virtual testing of cell tractions using numerical or theoretical models could be a potential tool for data integration (Lemmon and Romer 2010; Oers et al. 2014; Vermolen and Gefen 2015) and has demonstrated efficiency to further interpolate TFM measurements. Zielinski et al. (2013) simulated two-dimensional (2D) and three-dimensional (3D) cell-ECM interactions to quantify the sensitivity of traction force to parameters including cell stiffness, focal adhesion density, cell aspect ratio, and contractility. The cell contraction was modeled as the inward pressure. The cell stiffness and focal adhesion density were identified as two major parameters affecting the traction forces. Oakes et al. (2014) have proposed a physical model for adherent cells, considering contraction as a uniform inward pressure combined with a uniform boundary line tension. They observed that cell shape influenced the traction force distribution, and that cellular strain energy density exhibited minimal alternations in response to different substrates. Edwards and Schwarz (2011) have modeled cell contraction as thermal elasticity, in which temperature alternations induced cell contraction. It was then shown that traction forces concentrated around the cell periphery, especially along the stiffer direction of ECM. However, there is little data to quantify cellular tensional homeostasis.

To identify key index factors for maintaining cellular tensional homeostasis, a numerical model was developed in this work to mimic TFM measurements and capture the detailed cell-ECM interactions. Contraction was modeled as eigenstrain, which could induce isometric cell contraction without external forces and better match with cell physiology. We hypothesized that a cell strives to maintain its pre-set eigenstrain in response to mechanical disturbance. Numerical models were used to integrate the published experimental observations in 3 T3 fibroblasts (Oakes et al. 2014) as well as our TFM results for bovine aortic endothelial cells (BAECs). The predicted cell mechanics were used to test our hypothesis. This work could help to advance the understanding of cell-ECM interactions, specifically cell mechanosensing, and may lead to better regenerative strategies. 


\section{Materials and methods}

\subsection{TFM measurements of BAECs}

BAECs were maintained at $37{ }^{\circ} \mathrm{C}$ and $5 \% \mathrm{CO}_{2}$ in Medium 199 (Invitrogen) supplemented with $10 \%$ FetalClone III (HyClone), and $1 \%$ each of penicillin-streptomycin (Invitrogen), MEM amino acids (Invitrogen) and MEM vitamins (Invitrogen). Variably compliant polyacrylamide (PA) gels coated with $0.1 \mathrm{mg} / \mathrm{mL}$ rat tail type I collagen (BD Biosciences) were prepared by altering the ratio of acrylamide to bis-acrylamide (BioRad) in the polymerization solution as described previously (Califano and Reinhart-King 2008; Wang and Pelham 1998). Gels were synthesized with Young's moduli of 1 and $10 \mathrm{kPa}$ to mimic physiologically relevant tissue stiffness (Engler et al. 2004). Cells were seeded on PA gels embedded with $0.5 \mu \mathrm{m}$ fluorescent beads (Invitrogen) and allowed to attach and spread overnight. Bead fields were imaged using a Zeiss Axio Observer.Z1m microscope with a Hamamatsu ORCAER camera using a $20 \times$ objective before (stressed configuration) and after removal (relaxed configuration) of the cells with trypsin (Invitrogen). These images allowed substrate displacements to be tracked with correlation-based optical flow (Marganski et al. 2003). Substrate displacements were then translated into a strain field that was used to compute the traction strain using Bayesian statistics that maximized the most likely traction field describing a given strain field. The substrate strains were converted to traction stresses using the LIBTRC analysis library developed by Professor Micah Dembo of Boston University (Dembo and Wang 1999).

\subsection{Numerical modeling}

Numerical models were developed to mimic the TFM measurements of both BAECs and 3 T3 fibroblasts cultured on substrates of various stiffnesses (Fig. 1a). For shape-controlled 3T3 fibroblasts (Oakes et al. 2014), the round cell had a diameter of $30 \mu \mathrm{m}$ and a height of 5 $\mu \mathrm{m}$. The substrate was assumed as a cuboid $(75 \times 75 \times 5 \mu \mathrm{m})$ with a fixed bottom surface. A total of 205 focal adhesions were generated to connect the cellular bottom with the top surface of the substrate, excluding the nucleus area which is considered $10 \mu \mathrm{m}$ in diameter (Milo and Phillips 2015). The focal adhesions were modeled as truss 
(a)
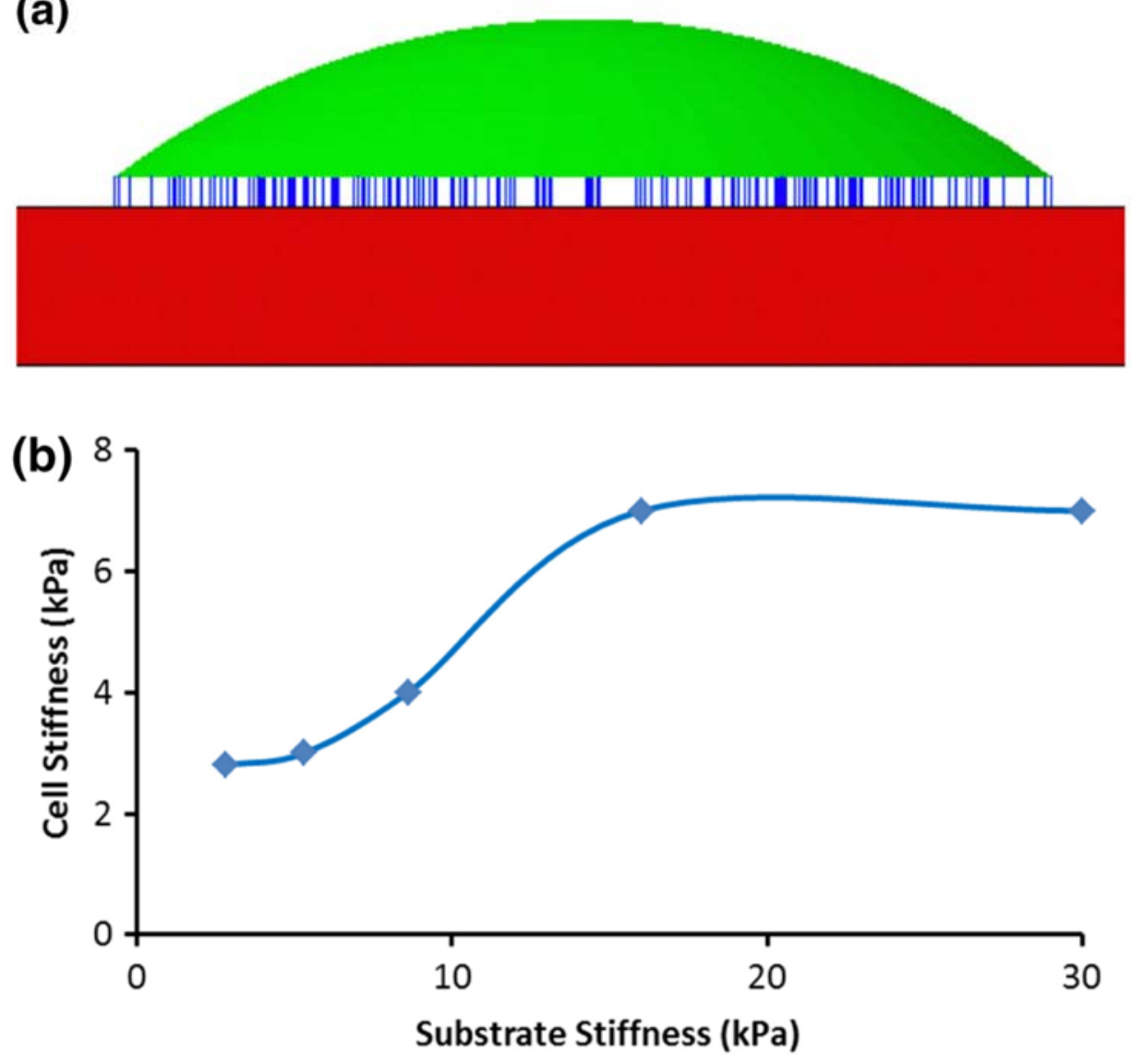

Fig. 1. a) Three-dimensional model of cell-substrate interactions; b) adherent cell stiffness in response to varied substrate stiffness.

elements with a length of $1 \mu \mathrm{m}$ (Franz and Müller 2005) and a stiffness of $20 \mathrm{nN} / \mu \mathrm{m}$. The cells were modeled as an isotropic elastic material with variable stiffness in response to substrate stiffness, which was adopted from measurements by Solon et al. (2007), as depicted in Fig. 1b. Specifically, cell stiffness increased with a stiffer substrate and reached a plateau as the substrate stiffness exceeded $16 \mathrm{kPa}$. The constitutive model for linear isotropic materials was represented by the generalized Hooke's law that relates the Cauchy stress tensor $\left(\sigma_{i j}\right)$ and the infinitesimal strain tensor $\left(\varepsilon_{i j}\right)$ as

$$
\varepsilon_{i j}=\frac{1+v}{E} \sigma_{i j}-\frac{v}{E} \sigma_{k k} \delta_{i j}
$$

where $\delta_{i j}$ is the Kronecker delta, $E$ is Young's modulus and $v$ is Poisson's ratio. 
For BAECs, the cell geometry was reconstructed from corresponding TFM images in ImageJ software ( $\mathrm{NIH}$, Bethesda, MD). The cell stiffness was reverse-fitted as 1.5 and $3 \mathrm{kPa}$ in response to substrate stiffness of 1 and $10 \mathrm{kPa}$, respectively, in order to match the traction stress field measured by TFM.

Cell contractility was modeled as eigenstrain, which was imposed to induce isometric contraction as the initial condition (Edwards and Schwarz 2011; Pan and Zhong 2016). The eigenstrain was defined as any nonelastic strains in the material without any external forces, such as strains caused by thermal expansion mismatch or phase transformation, plastic strains, etc. (Jun and Korsunsky 2010). In this work, the eigenstrain was the contraction strain of a cell. If a cell is free to contract, i.e., an isolated cell without constraints from the environment, this leads to zero stress in the cell. In reality, each cell is constrained by its local mechanical environment, and this leads to tensional stress within the cell as well as traction stress exerted against the ECM. In this work, a -20\% eigenstrain was imposed on the cell, which was reverse-fitted from the published experimental data (Oakes et al. 2014) to match the measured traction stresses. The strain energy on the substrate was calculated (Butler et al. 2002) as

$$
W=1 / 2 \int \mathbf{T}(\mathbf{r}) \cdot \mathbf{u}_{s}(\mathbf{r}) \mathrm{d} A
$$

where $\mathbf{T}(\mathbf{r})$ is the cell contraction-induced traction stress on the substrate, $\mathbf{u}_{s}(\mathbf{r})$ is displacement, and $A$ is the targeted area.

\subsection{BAEC cytoskeletal visualization}

BAECs were allowed to adhere and spread overnight on 1 and $10 \mathrm{kPa}$ polyacrylamide gels. Cells were then fixed with $3.7 \%$ formaldehyde (VWR) and permeabilized using 1\% triton (VWR), followed by blocking with $1 \%$ bovine serum albumin (Sigma) and incubation with Alexa Fluor 488 Phalloidin (Life Technologies) to visualize F-actin. Cells were imaged using a Zeiss Axio Observer.Z1m microscope with a Hamamatsu ORCA-ER camera using a $40 \times$ oil immersion objective. 


\section{Results}

Our model was used to capture the published experimental results for 3 T3 fibroblasts (Oakes et al. 2014). Briefly, the round cell shape was controlled to decouple it from the effect of substrate stiffness on cell geometry. The substrate mechanics were then characterized in terms of traction stress $(\mathrm{kPa})$ and displacement (magnitude, $\mu \mathrm{m})$, as well as the average strain energy $(\mathrm{pJ})$. The corresponding modeling results for substrate stiffnesses ranging from 2.8 to $30 \mathrm{kPa}$ are shown in Fig. 2. Using an eigenstrain-induced cell contraction, a good match was observed between our model predictions and the published experimental and numerical data (Oakes et al. 2014). Moreover, our model prediction demonstrated a better fit than the existing numerical model (Oakes et al. 2014). Increased displacement and traction stress were found around cell edges. The substrate traction

(a)
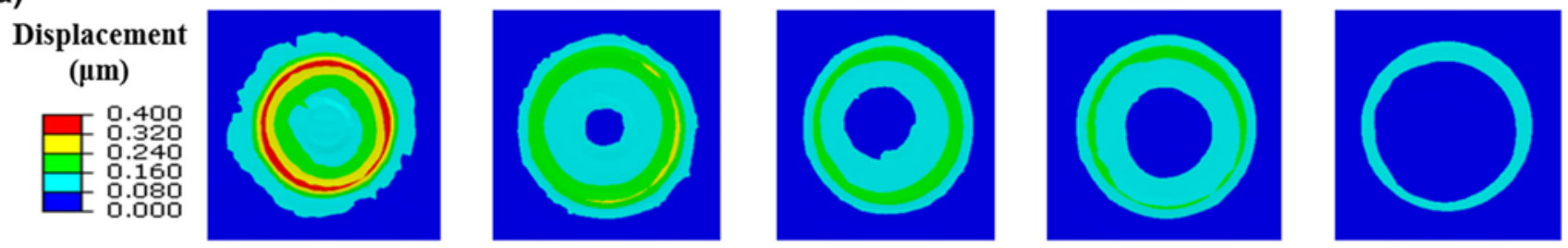

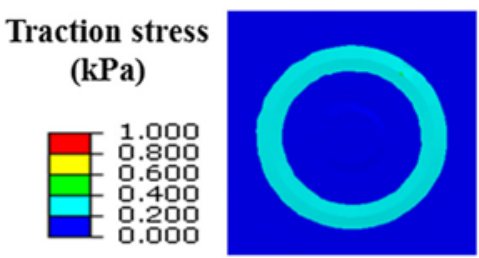

Stiffness

$2.8 \mathrm{kPa}$

(b)

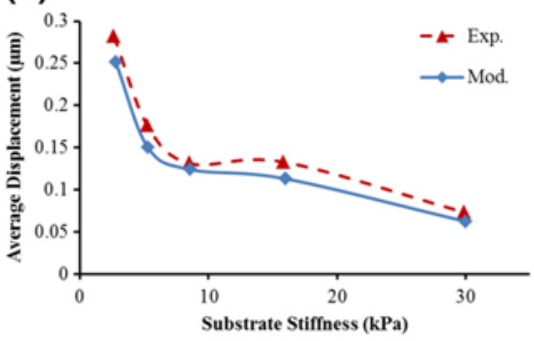

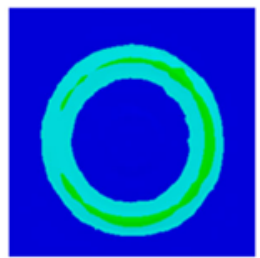

$5.3 \mathrm{kPa}$

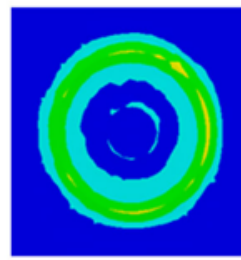

$8.6 \mathrm{kPa}$

(c)

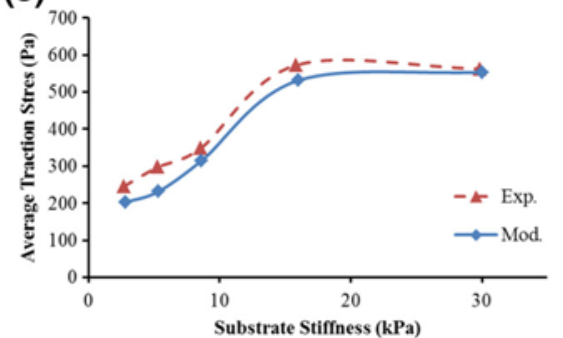

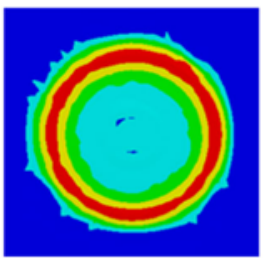

$16 \mathrm{kPa}$

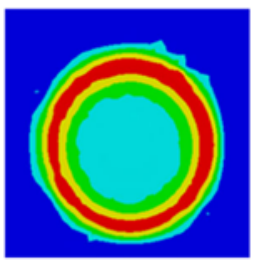

$30 \mathrm{kPa}$

(d)

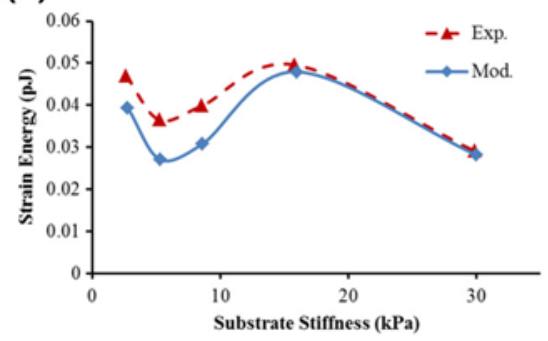

Fig. 2. Cell-induced substrate mechanics depend on substrate stiffness. a) Substrate displacement and traction stressmaps obtained from numerical models; b-d) average substrate displacement, traction stress and strain energy are predicted by numerical models and compared to the published experimental data. Reproduced with permission from Oakes et al. (2014). 
stress and displacement were inversely related to an increase in substrate stiffness. Specifically, the same eigenstrain contraction of cells led to a larger displacement with lower stresses on soft substrates, compared to stiff ones (Fig. 2a). Furthermore, we quantified the average displacement, average traction stress, and strain energy exerted on the substrate (Fig. $2 \mathrm{~b}-\mathrm{d}$ ). A substrate stiffness below $10 \mathrm{kPa}$ led to rapid changes in both substrate traction stress and displacement. This trend plateaued on stiffer substrates. Interestingly, the effect of substrate stiffness on strain energy showed an oscillating pattern. This could be explained by the rapid shift between the average displacements and traction stresses.

Cell mechanics under varied substrate stiffness were further investigated (Fig. 3). In response to increased substrate stiffness, the average cell stress followed the same trend as the substrate traction stress depicted in Fig. 2c. Notably, the average maximum principal strain of the cell had minimal sensitivity to the substrate stiffness. As the substrate stiffness varied between 2.8 and $30 \mathrm{kPa}$, the average maximum principal strain of the cell was reduced by $7.8 \%$ (from 14.1 to $13.0 \%$ ), compared to a $12.5 \%$ reduction assuming a constant cell stiffness independent of substrate rigidity. This indicated that dynamic tuning of cell stiffness in response to the mechanical environment is used to maintain a cell average strain.

Our hypothesis based on eigenstrain as the cellular set-point was also tested using BAECs with uncontrolled morphologies. Representative substrate traction stress maps (Fig. 4) demonstrated that peak

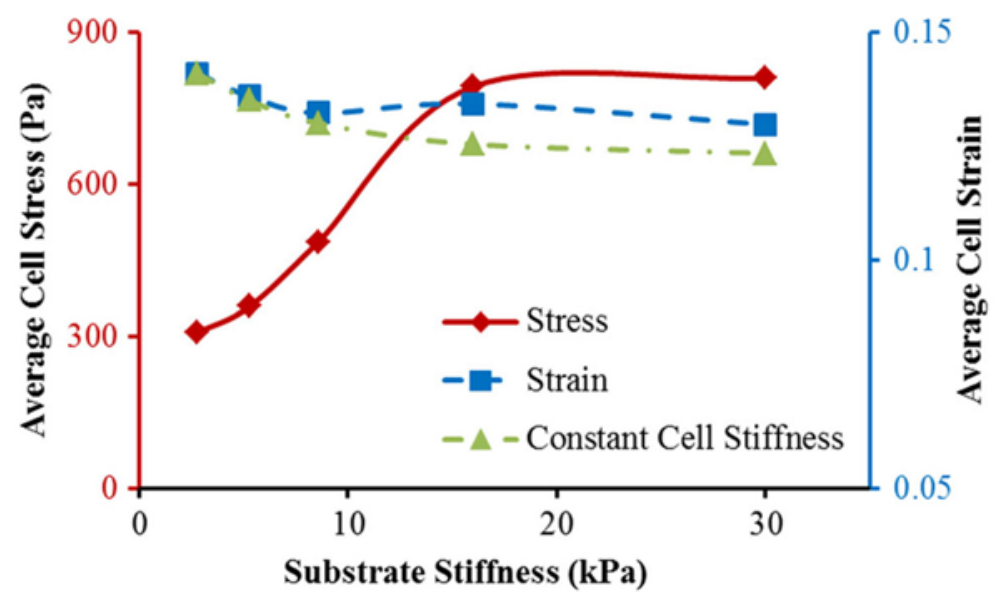

Fig. 3. Substrate stiffness altered the average stress in a single cell, while its average maximal principal strain remained constant. 

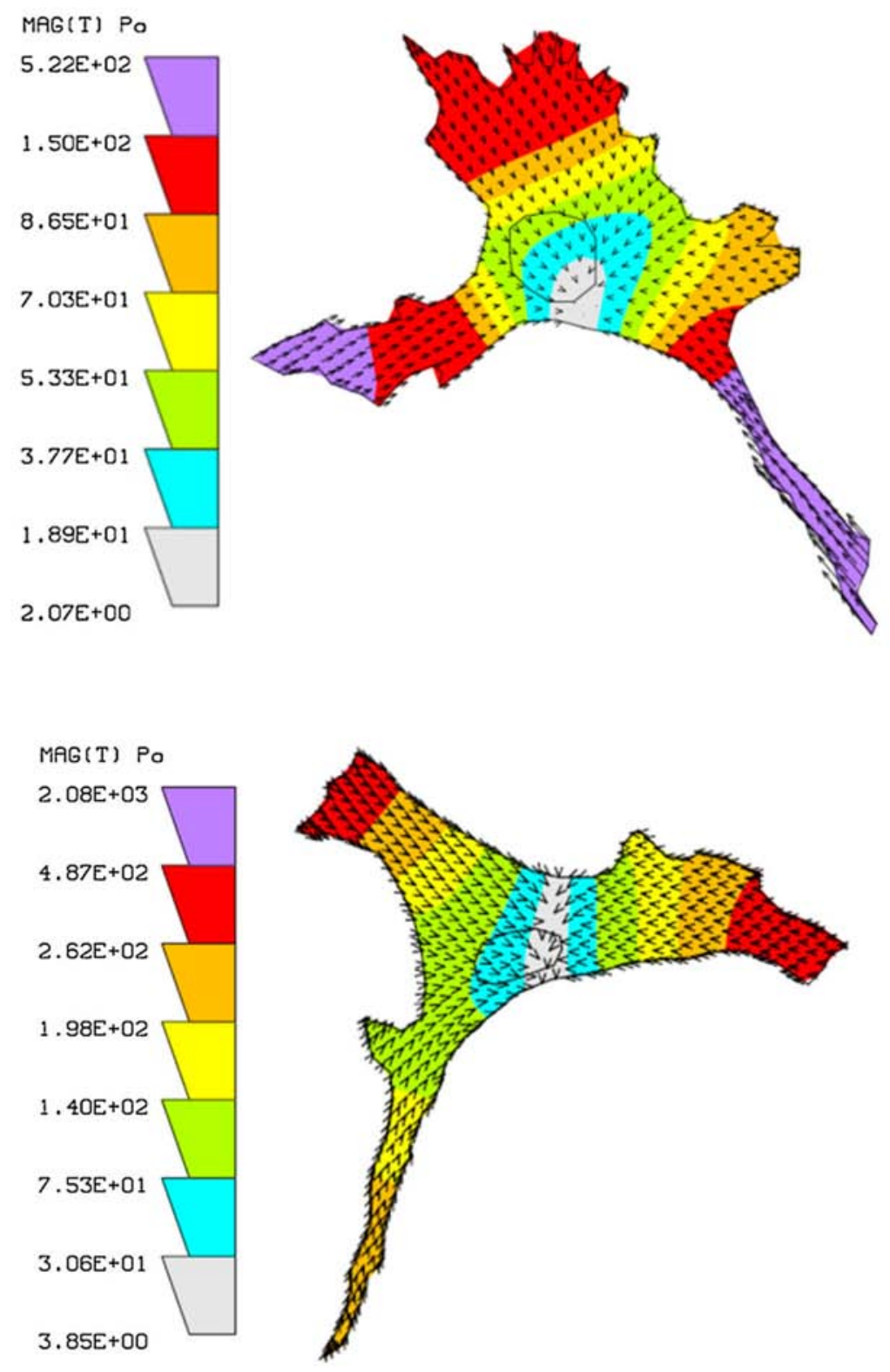

Fig. 4. Representative traction stress map $(\mathrm{kPa})$ for $\mathrm{BAECs}$ cultured on the substrate with a stiffness of $1 \mathrm{kPa}$ (upper) and $10 \mathrm{kPa}$ (lower), respectively.

stress is located at cell protrusions and is distant from the central nucleus area. Similar stress patterns were predicted from our computational models, which were reconstructed from TFM images of individual cells assuming a $-20 \%$ eigenstrain (Fig. 5a). However, the peak substrate stress obtained from our simulations was generally less than what was measured using TFM. As the substrate stiffness changed 
(a)

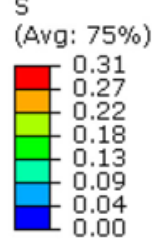

(b)
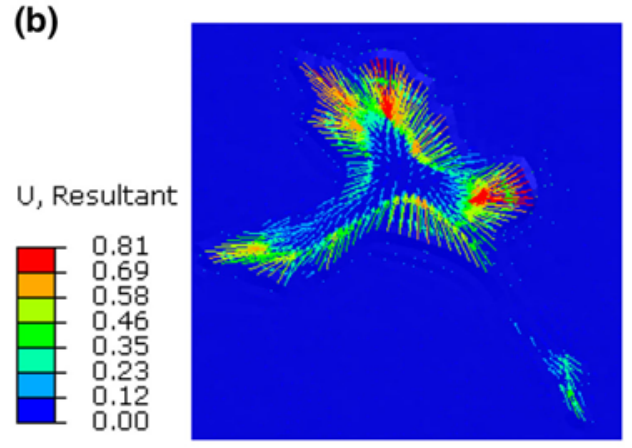

(c)

$\mathrm{U}$, Resultant
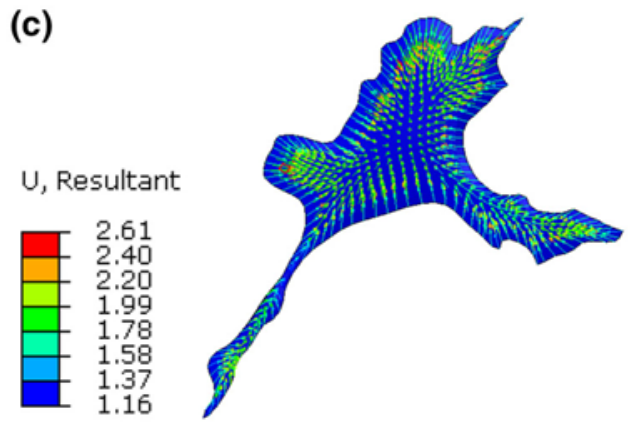

(d)
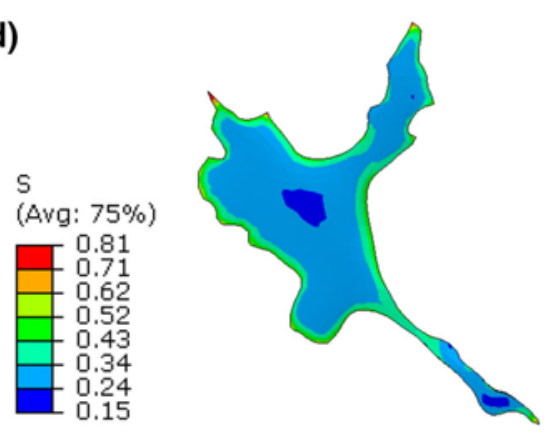

U, Resultant

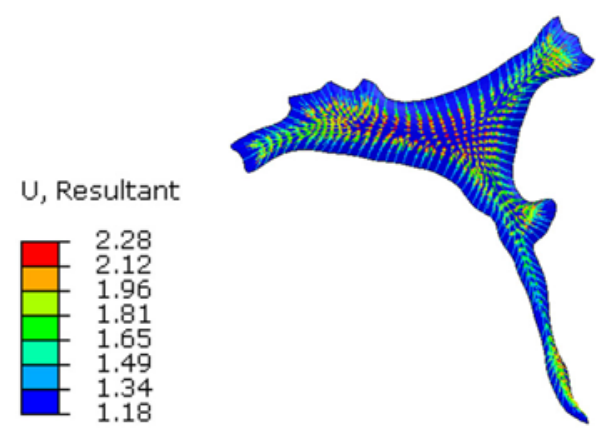

$\mathrm{U}$, Resultant
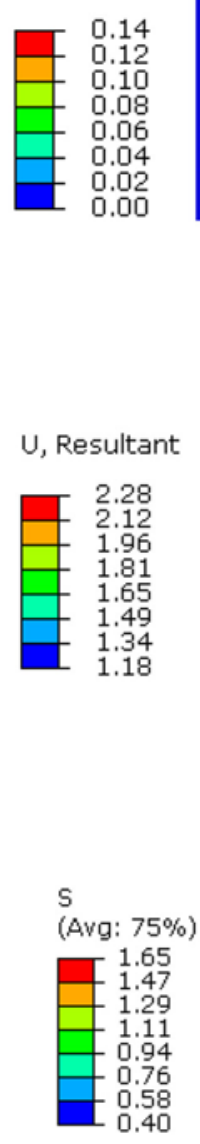

Fig. 5. Representative model predictions for BAECs cultured on substrates with a stiffness of $1 \mathrm{kPa}$ (left) and $10 \mathrm{kPa}$ (right): a) substrate traction stress map ( $\mathrm{kPa}$ ), b) substrate displacement map $(\mu \mathrm{m})$, c) cell displacement map $(\mu \mathrm{m})$, d) cell stress map $(\mathrm{kPa})$. 
from 1 to $10 \mathrm{kPa}$, peak substrate stresses of 0.31 and $0.57 \mathrm{kPa}$, respectively, were obtained from our simulations, compared to 0.52 and 2 $\mathrm{kPa}$ measured using TFM. Our computational model allowed us to probe the displacement or stress virtually anywhere within the computational domain, a feature that was not affected by experimental techniques. Specifically, Fig. 5b illustrates the cell contraction-induced substrate displacement map using our model. Figure $5 c$, d depicts the cellular response in terms of cell stress and displacement. It is clear that a stiffer substrate leads to higher stresses as well as decreased cell and substrate displacements (Fig. 5). The simulated integrin attachments between cells and their substrate resulted in larger cellular stresses and displacements than the substrate stresses and displacements. Table 1 summarizes both experimental results and the computational predictions. The predicted average substrate stress is within the range of the experimental measurements. It is worth noting that the change in the average cell strain was minimal compared to other parameters, such as stress, displacement, stiffness, or spread area. By comparing the cell average strain magnitudes between BAECs and fibroblast cells (Fig. 3), it is interesting to observe that the cell average strain is different for both cell types, but the cell contraction in terms of eigenstrain is the same.

\section{Discussion}

In this work, cell-ECM interactions were examined through combined TFM measurements and numerical models to quantify the tensional homeostasis of cells. The working hypothesis is that cellular behaviors on various substrates are regulated by the contraction strain of cells.

Table 1. Characteristics of bovine aortic endothelial cells from both TFM measurements (labeled) and numerical models.

\begin{tabular}{lcc}
\hline Substrate stiffness $(\mathrm{kPa})$ & 1 & 10 \\
Cell stiffness $(\mathrm{kPa})$ & 1.5 & 3 \\
Cell spread area $(\mathrm{TFM})\left(\mu \mathrm{m}^{2}\right)$ & $1600 \pm 148$ & $1693.7 \pm 283$ \\
Average stress on substrate $(\mathrm{TFM})(\mathrm{Pa})$ & $81.3 \pm 20.0$ & $189.6 \pm 23.7$ \\
Average stress on substrate $(\mathrm{Pa})$ & $92.0 \pm 5.5$ & $208.9 \pm 21.8$ \\
Cell average stress $(\mathrm{Pa})$ & $228 \pm 9$ & $559 \pm 23$ \\
Cell average strain & $5 \% \pm 1.1 \%$ & $3.73 \pm 0.2 \%$ \\
\hline
\end{tabular}


A $-20 \%$ eigenstrain was implemented to mimic the cell contraction on various substrates. This technique was validated in previous literature (Edwards and Schwarz 2011). Our TFM measurements of BAECs, as well as published TFM observations in 3 T3 fibroblasts (Oakes et al. 2014), were used to validate the numerical model. Our results indicate that eigenstrain, an efficient set-point for cell contraction, regulates cell behaviors in response to various mechanical stimuli.

The motivation to determine the set-point of a cell went back to the work by Brown et al. (1998). They quantified how fibroblasts, cultured within three-dimensional collagen lattices, responded to predefined static and dynamic tensional loadings on the gel. The data indicated that increased loading led to an acute reduction in cell contractile force, and vice versa. However, after unloading, the gel embedded with living fibroblast cells maintained an equilibrium tensional force of approximately 40-60 dynes/million cells. This indicated that tensional force correlated with the magnitude of the external stimuli, instead of its loading history. At each loading level, the tensional force was the same. Reflected in our eigenstrain model, different loading magnitudes correspond to the different substrate stiffnesses. The tensional force at discrete substrate stiffness remained at the same level. Mizutani et al. (2004) investigated the same problem from the perspective of altered cell stiffness. They observed that external substrate stretch caused instantaneous cell stiffness alterations and fibroblasts returned to their initial cell stiffness after unloading. These data demonstrated that mechanical stimuli induced acute changes in cell stiffness that correlated with the magnitude of the applied stimuli. In addition, this indicated that our numerical model should consider the stiffness adaptation of cells to different substrates. Overall these observations illustrated that the mechanical behaviors of cells are correlated with the magnitude of mechanical stimuli they experience, instead of the loading history. However, the relationship between different levels of mechanical stimuli, including different substrate or gel stiffnesses, was not identified, which is the basis we used to identify the set-point of cells, i.e., tensional homeostasis.

Our eigenstrain model captured TFM results of 3T3 fibroblasts in terms of substrate displacement and traction stress maps. It is interesting to note that our model prediction of average substrate displacement, traction stress, and strain energy has demonstrated a better match than the published numerical model. In the literature (Oakes 
et al. 2014), cell contraction was simulated as a contractile pressure of $2.4 \mathrm{kPa}$ along with a uniform membrane tension of $0.7 \mathrm{nN} / \mu \mathrm{m}$, compared to our model implementation using an eigenstrain of $-20 \%$. In addition, cell stiffness in the published model was assumed to be constant, whereas we adopted the measured acute cell stiffness, which varied in response to different substrate stiffnesses (Solon et al. 2007). The stiffness adaptation of cells correlated well with the observations that cells reorganize their actin cytoskeleton into bundles or stress fibers on stiff substrates (Solon et al. 2007; Yeung et al. 2005; Parker et al. 2002; Théry et al. 2006). The above-mentioned differences in numerical models led to different conclusions about the regulatory mechanisms of cells. For example, Oakes et al. (2014) found that the strain energy of a cell was correlated with the cell spread area, and the local curvature regulated the traction stress distributions on the substrate. Our main conclusion is that cells exhibit a constant strain regardless of altered substrate deformation or traction stress distributions in response to varied substrate stiffness. The eigenstrain and stiffness adaptations were two key factors that improved the performance of our model.

TFM measurements of BAECs demonstrated that traction stresses and cell area vary in response to substrate stiffness. The predicted traction stress distributions with higher stress located at cell protrusions (Fig. 5) agree with our TFM data as well as prior published TFM results (Edwards and Schwarz 2011). Our validated model allowed us to further investigate the cellular responses in different mechanical environments. Our model indicated that cells exhibit larger stresses and smaller displacements when cultured on a stiffer matrix, and followed the same trend as the TFM data when substrate mechanics were varied. But the predicted cellular displacements and stresses were found to be larger than the substrate displacements and stresses. This could be explained by the existence of focal adhesions. The peak substrate stress obtained from our simulations was relatively less than what was measured using TFM. This could be attributed to subcellular heterogeneity, as evidenced by the actin cytoskeleton from our BAEC fluorescent images (Fig. 6). BAECs reorganize their cytoskeleton in response to substrate stiffness, indicating alterations in cell stiffness. In our numerical model, we considered bulk cell stiffening but not the inhomogeneity within the cell. The homogeneous cell simplification will alter the traction stress localization, but will not impact our understanding 

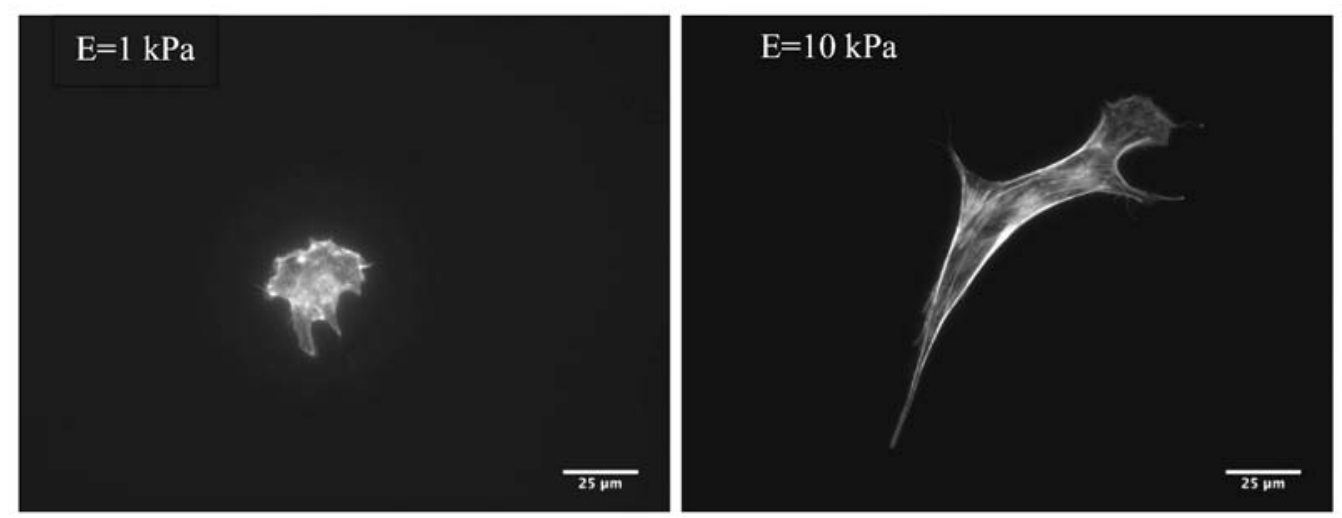

Fig. 6. Representative cytoskeletal maps of BAECs cultured on the substrate with a stiffness of 1 and $10 \mathrm{kPa}$.

of the tensional homeostasis of a cell. For unconstrained BAECs, we demonstrated that the cell average strain was constant regardless of cell morphology (Table 1). Moreover, the cell average strain in BAECs was less than half of the strain in fibroblast cells. This indicated that the cell average strain is cell type-specific. However, it is worth noting that the eigenstrains on both cells are the same. This suggests that different cell types have the same set-point level in terms of cell contraction. Further experimental evidence for additional cell types is needed to validate this observation.

The practical implication of this work is the identification of cellular strain as the tensional homeostasis state that each cell type maintains following mechanical disturbances, including alteration of substrate stiffness. Understanding the tensional homeostasis of cells is an important consideration in cellular biophysics and could provide a starting point for investigating many biological processes such as cell growth, differentiation, and migration. We found that cellular strain was independent of a cell's mechanical environment, and speculate that eigenstrain may be a cellular set-point that is conserved across cell types. This needs to be further tested through experimental measurements that include the cell morphology and the mechanics of focal adhesions. Measurements of contractility may be useful for monitoring cell mechanosensing as well as dynamic ECM remodeling. This work has the potential to elucidate why cells change their area and morphologies in response to substrate stiffness. Our mechanical model, based on the cell shape with the assumption of constant focal adhesion density, is a significant first step in quantifying the 
tensional homeostasis of cells. The dynamical behaviors of focal adhesions might play a role in the cellular tensional hemostasis, which could be incorporated in our future work. The relevant cellular remodeling processes can also be addressed in future studies by incorporating the dynamic reorganization of cytoskeletal architecture and focal adhesion dynamics that occur when cells interact with their ECM (Discher et al. 2005; Kumar et al. 2016; Humphrey et al. 2014). In summary, our finding that eigenstrain is consistent could help to advance the understanding of the cell-ECM relationship, specifically with respect to cell mechanosensing, and may lead to better regenerative strategies (Chawla 2012; Zhong et al. 2007).

Acknowledgments - This work was supported in part by the National Science Foundation CAREER award (CBET-1254095) and the Foundation of Fujian Educational Committee (JAT170422). The authors also thank Mr. Hozhabr Mozafari for constructive comments.

Conflict of interest - The authors have no conflicts of interest.

\section{References}

Beloussov L, Louchinskaia N, Stein A (2000) Tension-dependent collective cell movements in the early gastrula ectoderm of Xenopus laevis embryos. Dev Genes Evol 210(2):92-104

Brown R et al (1998) Tensional homeostasis in dermal fibroblasts: mechanical responses to mechanical loading in three-dimensional substrates. J Cell Physiol 175(3):323-332

Butler JP et al (2002) Traction fields, moments, and strain energy that cells exert on their surroundings. Am J Physiol Cell Physiol 282(3):C595-C605

Califano JP, Reinhart-King CA (2008) A balance of substrate mechanics and matrix chemistry regulates endothelial cell network assembly. Cell Mol Bioeng 1(2-3):122-132

Califano JP, Reinhart-King CA (2010) Substrate stiffness and cell area predict cellular traction stresses in single cells and cells in contact. Cell Mol Bioeng 3(1):68-75

Chawla K (2012) Biomaterials for tissue engineering and regenerative medicine: Treatment of musculoskeletal injury and disease. Mater Sci Eng A 557:45-53

Dembo M, Wang Y-L (1999) Stresses at the cell-to-substrate interface during locomotion of fibroblasts. Biophys J 76(4):2307-2316

Discher DE, Janmey P, Wang Y-L (2005) Tissue cells feel and respond to the stiffness of their substrate. Science 310(5751):1139-1143 
Edwards CM, Schwarz US (2011) Force localization in contracting cell layers. Phys Rev Lett 107(12):128101

Engler AJ et al (2004) Surface probe measurements of the elasticity of sectioned tissue, thin gels and polyelectrolyte multilayer films: correlations between substrate stiffness and cell adhesion. Surf Sci 570(1):142-154

Franz CM, Müller DJ (2005) Analyzing focal adhesion structure by atomic force microscopy. J Cell Sci 118(22):5315-5323

Hadjipanayi E, Mudera V, Brown RA (2009) Guiding cell migration in 3D: a collagen matrix with graded directional stiffness. Cell Motil cytoskelet 66(3):121-128

Humphrey JD, Dufresne ER, Schwartz MA (2014) Mechanotransduction and extracellular matrix homeostasis. Nat Rev Mol Cell Biol 15(12):802-812

Jun T-S, Korsunsky AM (2010) Evaluation of residual stresses and strains using the eigenstrain reconstruction method. Int J Solids Struct 47(13):1678-1686

Kumar A et al (2016) Talin tension sensor reveals novel features of focal adhesion force transmission and mechanosensitivity. J Cell Biol 213(3):371-383

Lemmon CA, Romer LH (2010) A predictive model of cell traction forces based on cell geometry. Biophys J 99(9):L78-L80

Lin S et al (2017) Active stiffening of F-actin network dominated by structural transition of actin filaments into bundles. Compos Part B Eng 116:377-381

Marganski WA, Dembo M, Wang Y-L (2003) Measurements of cell-generated deformations on flexible substrata using correlation-based optical flow. Methods Enzymol 361:197-211

Milo R, Phillips R (2015) Cell biology by the numbers. Garland Science, New York

Mizutani T, Haga H, Kawabata K (2004) Cellular stiffness response to external deformation: tensional homeostasis in a single fibroblast. Cell Motil Cytoskelet 59(4):242-248

Munevar S, Wang Y-L, Dembo M (2001) Traction force microscopy of migrating normal and H-ras transformed 3T3 fibroblasts. Biophys J 80(4):1744-1757

Oakes PW et al (2014) Geometry regulates traction stresses in adherent cells. Biophys J 107(4):825-833

Oria $\mathrm{R}$ et al (2017) Force loading explains spatial sensing of ligands by cells. Nature 552(7684):219

Pan Y, Zhong Z (2016) Micromechanical modeling of the wood cell wall considering moisture absorption. Compos Part B Eng 91:27-35

Parker KK et al (2002) Directional control of lamellipodia extension by constraining cell shape and orienting cell tractional forces. FASEB J 16(10):1195-1204

Paszek MJ et al (2005) Tensional homeostasis and the malignant phenotype. Cancer Cell 8(3):241-254

Prager-Khoutorsky $\mathrm{M}$ et al (2011) Fibroblast polarization is a matrix-rigidity-dependent process controlled by focal adhesion mechanosensing. Nat Cell Biol 13(12):1457-1465

Rape $A D$, Guo W-H, Wang Y-L (2011) The regulation of traction force in relation to cell shape and focal adhesions. Biomaterials 32(8):2043-2051 
Rittweger J et al (2009) Bone loss in the lower leg during 35 days of bed rest is predominantly from the cortical compartment. Bone 44(4):612-618

Solon J et al (2007) Fibroblast adaptation and stiffness matching to soft elastic substrates. Biophys J 93(12):4453-4461

Tan JL et al (2003) Cells lying on a bed of microneedles: an approach to isolate mechanical force. Proc Natl Acad Sci 100(4):1484-1489

Théry M et al (2006) Anisotropy of cell adhesive microenvironment governs cell internal organization and orientation of polarity. Proc Natl Acad Sci 103(52):19771-19776

van Oers RF et al (2014) Mechanical cell-matrix feedback explains pairwise and collective endothelial cell behavior in vitro. PLoS Comput Biol 10(8):e1003774

Vermolen F, Gefen A (2015) Semi-stochastic cell-level computational modelling of cellular forces: application to contractures in burns and cyclic loading. Biomech Model Mechanobiol 14(6):1181-1195

Wang Y-L, Pelham RJ (1998) Preparation of a flexible, porous polyacrylamide substrate for mechanical studies of cultured cells. Methods Enzymol 298:489-496 